Research Paper

\title{
Economic Impact of Prosthetic Joint Infection - an Evaluation Within the Portuguese National Health System
}

\author{
Arnaldo Sousa ${ }^{1 凶}$, André Carvalho ${ }^{1}$, Cláudia Pereira ${ }^{2 *}$, Ernestina Reis ${ }^{2 *}$, Ana Cláudia Santos ${ }^{3^{*}}$, Miguel \\ Abreu $^{4^{*}}$, Daniel Soares ${ }^{1^{*}}$, Ricardo Fragoso ${ }^{5}$, Susana Ferreira ${ }^{6}$, Marcio Reis ${ }^{7}$, Ricardo Sousa ${ }^{*}$ \\ 1. Department of Orthopedics, Centro Hospitalar do Porto, Porto, Portugal \\ 2. Department of Internal Medicine, Centro Hospitalar do Porto, Porto, Portugal \\ 3. Department of Microbiology, Centro Hospitalar do Porto - Hospital de Santo António, Porto, Portugal \\ 4. Department of Infectious Diseases, Centro Hospitalar do Porto, Porto, Portugal \\ 5. Department of Information Systems, Centro Hospitalar do Porto, Porto, Portugal \\ 6. Department of Information Management, Centro Hospitalar do Porto, Porto, Portugal \\ 7. Orthopedics Department Manager, Centro Hospitalar do Porto, Porto, Portugal \\ * Porto Bone and Joint Infection Group (GRIP), Centro Hospitalar do Porto, Portugal \\ $\triangle$ Corresponding author: Arnaldo Sousa. Email: arnaldosousa5@hotmail.com; Department of Orthopedics, Centro Hospitalar do Porto - Hospital de Santo \\ António, Largo Professor Abel Salazar, 4099-001 Porto, Portugal. Tel. (00351)222 077500 - Fax. (00351)222 087632 \\ (C) Ivyspring International Publisher. This is an open access article distributed under the terms of the Creative Commons Attribution (CC BY-NC) license \\ (https://creativecommons.org/licenses/by-nc/4.0/). See http://ivyspring.com/terms for full terms and conditions.
}

Received: 2018.07.13; Accepted: 2018.08.20; Published: 2018.09.08

\begin{abstract}
Introduction: Prosthetic infection is a devastating complication of arthroplasty and carries significant economic burden. The objective of this study was to analyze the economic impact of prosthetic hip and knee infection in Portuguese National Health System.

Material and Methods: Case-control study carried out from January 2014 to December 2015. The mean costs of primary arthroplasties and prosthetic revision surgeries for non-infectious reasons were compared with the costs of prosthetic infections treated with debridement and preservation of the prosthesis or with two-stage exchange arthroplasty. The reimbursement for these cases was also evaluated and compared with its real costs.

Results: A total of 715 primary arthroplasties, 35 aseptic revisions, 16 surgical debridements and 15 revisions for infectious reasons were evaluated. The cost of primary arthroplasties was 3,230€ in the hips and 3,618€ in the knees. The cost of aseptic revision was $6,089 €$ in the hips and 7,985€ in the knees. In the cases treated with debridement and implant retention the cost was 5,528€ in the hips and 4,009€ in the knees. In cases of infections treated with a two-stage revision the cost was $11,415 €$ and $13,793 €$ for hips and knees, respectively.

Conclusion: As far as we know this is the first study that analyzes the economic impact of prosthetic infection in the Portuguese context. Although direct compensation for treating infected cases is much lower than calculated costs, infected cases push the overall hospital case-mix-index upwards thus increasing financial compensation for the entire cohort of treated patients. This knowledge will allow for more informed decisions about health policies in the future.
\end{abstract}

Key words: prosthetic joint infection, Portugese National Health System

\section{Introduction}

Prosthetic join infection (PJI) is widely acknowledged as the most feared complication after total joint replacement. Despite modern preventive strategies, infection rates seem to be on the rise after total hip (THA) and knee (TKA) arthroplasty [1, 2].
Late infections occurring many years after index surgery are also becoming more common as the number of people living with some kind of total joint arthroplasty is increasing [3]. 
PJI is consistently associated with significant medical implications for the patient thus becoming a source of substantial burden not only for patients but also for hospitals and healthcare systems. As the requirements for total joint arthroplasty have been and are expected to steadily increase, so will the economic burden of infection $[4,5]$.

The real cost of treating an infected joint is not easy to ascertain. Naturally, it depends on a lot of variables such as the specific type of treatment, patient co-morbidities and even bacteria related factors such as antibiotic susceptibility profile. The full spectrum of economic impact comprises the more commonly reported direct in-hospital costs, but also outpatient direct costs (follow-up visits, rehabilitation, pharmacy, etc.) and even indirect costs that are virtually impossible to accurately assess, such as productivity loss, work absenteeism of the patient or even his caregivers.

Nevertheless, setting some kind of standard is critical to guide the rational use of existing economic resources and provide cost-effective care policies. Our primary goal is to provide baseline measurements of direct in-hospital costs of treating infection after total hip and knee arthroplasty in the Portuguese public health system and compare them with uneventful primary and aseptic revision arthroplasty. A secondary goal is to assess how these costs compare to current hospital reimbursement policy and its possible implications in the setting up of a reference center for the treatment of PJI.

\section{Material and Methods}

We conducted a retrospective case-control study at a single institution within the public Portuguese National Health System. We focused on a 2-year time period between January/2014 and December/2015. All hip or knee arthroplasty surgeries, either primary or revision, were identified on the institutional administrative electronic databases.

Cases were then separated into four different groups: 1) Uneventful primary surgery; 2) Aseptic revision surgery; 3) PJI treated with debridement, antibiotics and implant retention (DAIR) and; 4) PJI treated with two-stage revision surgery. The first two groups, where there was no infection, served as controls. All groups were also further subdivided into hip or knee arthroplasty.

Simple demographic and clinical data were obtained by reviewing electronic medical records. We also collected treatment related variables with impact to the final cost calculation such as the total length of stay and/or duration of stay in intensive care or orthopedic ward, as well as the duration of stay in the operating room.
Administrative and economic data was derived from institutional databases and was obtained by two independent non-medical researchers (RF and SF), that were blinded to the patients' diagnosis and outcome.

For our primary goal, the calculation of the total cost per episode of care was allocated into two main parts: Hospital Stay including costs during the patient's stay at the orthopedics department or intensive/intermediate care units, and Operating Room (OR) that includes all expenses made during surgery.

Hospital Stay includes costs with: 1) Staff - costs based on the duration of hospital stay and labor cost for each health professional; 2) Ward costs - indirect costs with maintenance, meals and accommodation; 3 ) Materials - average costs with clinical consumables; 4) Diagnostic tests - costs stipulated for imaging, microbiology, blood tests, etc.; 5) Medication pharmaceuticals dispatched separating Antibiotics.

Operating Room costs includes: 1) Staff - costs based on the duration of surgery and labor cost for each health professional involved; 2) OR costs - costs of equipment, structure and general and clinical support specific for each surgery; 3) Clinical Materials average costs with clinical consumables including the implants used.

With all these variables in mind, final costs were calculated for each case and average price for each of the previously differentiated groups are reported in Euros $(€)$.

With respect to our secondary goal, we analyzed together with the Orthopedic Department management the existing reimbursement methodology and rules in effect during the study period. A fixed price is payed for each inpatient treated regardless the patient specific diagnosis. This price is defined according to the available national health budget, with adjustments for the two major types of admissions (elective and emergency) and hospital classification within the National Health System (hospitals are classified regarding the dimension, scale, specialization and education). This fixed price is further multiplied by a factor of the hospital case-mix index (CMI). Each treated patient is given a complexity score (relative value) according to diagnosis related group (DRG) classification (All Patient Refined DRG). The hospital CMI takes into consideration all inpatients episodes of a hospital reflecting their diversity and clinical complexity. It can be used as hospital output measure and also to adjust the average cost per patient.

\section{Results}

In the study time period, we identified a total of 828 cases of hip or knee arthroplasty related surgeries. 
Patients who underwent tumor arthroplasties $(n=4)$, those whose main reason for hospital admission was not orthopedic $(n=2)$, as well as patients who had unrelated procedures or complications during the same hospitalization $(n=5)$ were excluded from the final analysis. We also excluded those in which we were not able to obtain the fundamental economic data $(n=36)$.

Ultimately, 781 patients were included in this study. In the first group of uneventful primary arthroplasties there were 715 patients (459 TKA, 256 THA). In the second group of revision surgery due to aseptic failure, 35 patients were studied (13 TKA, 22 THA). Every TKA revision surgery included in this study was complete, meaning both femoral and tibial parts were revised. However, regarding THA, only four cases had both femoral and acetabular components revised. For more accurate and fair comparison with infected cases, partial and complete revisions costs are discriminated.

Among the 31 PJI cases included, 16 patients (eight TKA and eight THA), were treated with debridement and implant retention and 15 patients (eight TKA and seven THA) were treated with a two-stage exchange protocol.

Basic demographic and clinical data of the cohort are detailed for TKA cases in table 1 and THA cases in table 2. There was no statistical difference between cases and controls with respect to age, gender proportion and ASA score.

Economic calculations are expressed in table 3 for knee cases and table 4 for hip cases. It is possible to observe that the average cost of uneventful primary arthroplasty was slightly higher for TKA $(3,618 €)$ compared to THA $(3,230 €)$.

The cost of treating an acute infection with debridements, antibiotics and implant retention, was on average $4,009 €$ for TKA and 5,528€ for THA. If we consider these together with the costs of primary joint replacement, infection in the early postoperative period more than doubles the cost of an uneventful joint replacement.

The average cost of revision knee surgery for aseptic reasons is $7,985 €$ which is around 2.2 times the cost of a primary TKA. On the other hand, the cost of aseptic revision hip surgery was $6,089 €$ considering all cases together and $7,840 €$ if we consider only the four cases where both components were revised. In summary, aseptic revision hip surgery costs around 1.9 to 2.4 times the cost of primary THA.

The total cost of two-stage exchange surgery for the treatment of PJI, considering both hospitalizations, is on average $13,793 €$ and $11,415 €$ for TKA and THA, respectively. This represents a cost of about 3.5 times the cost of uncomplicated primary TKA and
1.6 times the cost of revision TKA for aseptic reasons. Two-stage revision surgery in hips costs about 3.5 times as much as primary uncomplicated THA and 1.9 times as much as revision THA for aseptic reasons overall (roughly 1.5 times as much if we consider both components revisions only).

Table 1. Basic demographic and clinical data for 488 TKA patients

\begin{tabular}{lllll}
\hline & $\begin{array}{l}\text { Primary } \\
\text { Arthroplasty }\end{array}$ & $\begin{array}{l}\text { Aseptic } \\
\text { Revisions }\end{array}$ & DAIR & $\begin{array}{l}\text { Two-stage } \\
\text { Revision }\end{array}$ \\
\hline & $n=459$ & $n=13$ & $n=8$ & $n=8$ \\
\hline Age (years) & $68(47-88)$ & $68(58-80)$ & $69(62-76)$ & $68(39-77)$ \\
Female Gender & $372(81 \%)$ & $10(77 \%)$ & $5(63 \%)$ & $5(63 \%)$ \\
Length of Stay (days) & 6 & 7 & 13 & $23(14+9)$ \\
ICU stay (days) & 0.03 & 0.5 & 0.1 & 1.2 \\
Time in the OR (hours) & 2.24 & 2.6 & 4.29 & 7.9 \\
ASA score & 2.3 & 2.5 & 2.5 & 2.7 \\
\hline
\end{tabular}

DAIR - Debridement, Antibiotics and Implant Retention;

Table 2. Basic demographic and clinical data for 293 THA patients

\begin{tabular}{lllll}
\hline & $\begin{array}{l}\text { Primary } \\
\text { Arthroplasty }\end{array}$ & $\begin{array}{l}\text { Aseptic } \\
\text { Revisions }\end{array}$ & DAIR & $\begin{array}{l}\text { Two-stage } \\
\text { Revision }\end{array}$ \\
\hline & $n=256$ & $n=22(4+)$ & $n=8$ & $n=7$ \\
\hline Age (years) & $67(32-91)$ & $66(45-87)$ & $73(56-87)$ & $62(42-76)$ \\
Female Gender & $146(57 \%)$ & $10(45 \%)$ & $6(75 \%)$ & $3(43 \%)$ \\
Length of Stay (days) & 7 & 21 & 9 & $22(12+10)$ \\
ICU stay (days) & 0.45 & 0.7 & 0.2 & 2.1 \\
Time in the OR (hours) & 2.24 & 2.6 & 4.29 & 7.9 \\
ASA score & 2.4 & 2.6 & 2.5 & 2.8 \\
\hline
\end{tabular}

DAIR - Debridement, Antibiotics and Implant Retention;

+only 4 complete revisions

Table 3. Economic Data on 488 TKA cases (average cost per patient in euros)

\begin{tabular}{lllll}
\hline & $\begin{array}{l}\text { Primary } \\
\text { Arthroplasty } \\
(\mathrm{n}=459)\end{array}$ & $\begin{array}{l}\text { Aseptic } \\
\text { Revisions } \\
(\mathrm{n}=13)\end{array}$ & $\begin{array}{l}\text { DAIR } \\
(\mathrm{n}=\end{array}$ & $\begin{array}{l}\text { Two-stage } \\
\text { Revision } \\
(\mathrm{n}=8)\end{array}$ \\
\hline Total Costs & $\mathbf{3 , 6 1 8}$ & $\mathbf{7 , 9 8 5}$ & $\mathbf{4 , 0 0 9}$ & $\mathbf{1 3 , 7 9 3}$ \\
Total Hospital Stay & $\mathbf{1 , 0 2 4}$ & $\mathbf{1 , 2 8 9}$ & $\mathbf{2 , 6 7 7}$ & $\mathbf{4 , 3 8 8}$ \\
Staff & 521 & 405 & 1,120 & 1,944 \\
Ward costs & 262 & 151 & 135 & 1,141 \\
Materials & 78 & 409 & 661 & 252 \\
Diagnostic Tests & 62 & 126 & 78 & 433 \\
Medication & 83 & 94 & 78 & 108 \\
(excluding antibiotics) & & & & \\
Antibiotics & 4 & 19 & 553 & 239 \\
ICU stay & 14 & 85 & 52 & 271 \\
Total Operating Room & $\mathbf{2 , 5 9 4}$ & $\mathbf{6 , 6 9 6}$ & $\mathbf{1 , 3 3 2}$ & $\mathbf{9 , 4 0 5}$ \\
Staff & 277 & 306 & 204 & 621 \\
OR costs & 237 & 413 & 334 & 451 \\
Clinical Materials & 2,080 & 5,977 & 794 & 8,333 \\
(including prosthesis) & & & & \\
\hline
\end{tabular}

DAIR - Debridement, Antibiotics and Implant Retention

Table 5 presents the actual reimbursement for each of the four study groups. It is obvious that reimbursement is significantly lower than calculated costs for all groups. It covers $75 \%, 38 \%, 57 \%$ and $69 \%$ of the calculated costs for primary total joint arthroplasty, aseptic revision surgery, DAIR procedures and two-stage revision for infected cases respectively. The average loss per infected case varies between $2,068 €$ on average for DAIR cases and 7,515€ 
on average for two stage revision surgery. During the study's two-year period, total losses summed up $33,088 €$ for 16 DAIR cases and $112,575 €$ for 15 two-stage revision cases $(145,663 €$ for infected cases together).

Table 4. Economic Data on 293 THA cases (average cost per patient in euros)

\begin{tabular}{|c|c|c|c|c|}
\hline & $\begin{array}{l}\text { Primary } \\
\text { Arthroplasty } \\
(\mathrm{n}=256)\end{array}$ & $\begin{array}{l}\text { Aseptic } \\
\text { Revisions } \\
\left(\mathrm{n}=22\left[4^{*}\right]\right)\end{array}$ & $\begin{array}{l}\text { DAIR } \\
(\mathrm{n}= \\
8)\end{array}$ & $\begin{array}{l}\text { Two-stage } \\
\text { Revision } \\
(\mathrm{n}=7)\end{array}$ \\
\hline Total Costs & 3,230 & $6,089\left[7,840^{*}\right]$ & 5,528 & 11,415 \\
\hline Total Hospital Stay & 1,307 & $\mathbf{1 , 7 2 8}\left[1,739^{*}\right]$ & 3,923 & 4,584 \\
\hline Staff & 624 & 773 & 1,802 & 1,772 \\
\hline Ward costs & 407 & $151\left[162^{*}\right]$ & 291 & 1,026 \\
\hline Materials & 94 & 404 & 1,028 & 274 \\
\hline Diagnostic Tests & 95 & 248 & 317 & 703 \\
\hline $\begin{array}{l}\text { Medication } \\
\text { (excluding antibiotics) }\end{array}$ & 36 & 48 & 99 & 293 \\
\hline Antibiotics & 6 & 10 & 287 & 243 \\
\hline ICU stay & 45 & 94 & 87 & 273 \\
\hline Total Operating Room & 1,923 & $4,361\left[6,101^{*}\right]$ & 1,605 & 6,831 \\
\hline Staff & 238 & $305\left[351^{*}\right]$ & 304 & 464 \\
\hline OR costs & 292 & 392 & 208 & 719 \\
\hline $\begin{array}{l}\text { Clinical Materials } \\
\text { (including the prostesis) }\end{array}$ & 1393 & $3,664\left[5,358^{*}\right]$ & 1,093 & 5,648 \\
\hline
\end{tabular}

Table 5. Comparison between calculated costs and actual reimbursement for each group (TKA and THA considered together)

\begin{tabular}{lllll}
\hline & $\begin{array}{l}\text { Mean Actual } \\
\text { Reimbursement }\end{array}$ & $\begin{array}{l}\text { Reimbursement } \\
\text { vs Costs } \\
\text { proportion (\%) }\end{array}$ & $\begin{array}{l}\text { Mean } \\
\text { complexity } \\
\text { score }\end{array}$ & $\begin{array}{l}\text { Complexity Score } \\
\text { vs. Overall hospital } \\
\text { CMI (\%) }\end{array}$ \\
\hline $\begin{array}{l}\text { Primary } \\
\text { Joint }\end{array}$ & $2,623 €$ & $75 \%$ & 1.48 & $125 \%$ \\
$\begin{array}{l}\text { Replacement } \\
\text { Aseptic }\end{array}$ & $2,564 €$ & $38 \%$ & & \\
$\begin{array}{l}\text { Revisions } \\
\text { DAIR } \\
\text { procedures }\end{array}$ & $2,700 €$ & $57 \%$ & 2.09 & $177 \%$ \\
$\begin{array}{l}\text { Two-Stage } \\
\text { Exchanges }\end{array}$ & $5,168 €$ & $69 \%$ & 2.29 & $194 \%$ \\
\hline $\begin{array}{l}\text { DAIR - Debridement, Antibiotics and Implant Retention } \\
\text { CMI - case-mix index }\end{array}$ & & & \\
\end{tabular}

During the study time period, the overall hospital CMI was 1,2884 in 2014 and 1,0686 in 2015. Within this time period, there were 8,556 patients operated within the Orthopedic Department and overall department CMI was 1.25. We calculated the mean complexity score of the 16 DAIR cases contributed to a slight increase on the overall hospital CMI by 0.00033 in 2014 and 0.00012 in 2015, which lead to an increase in overall reimbursement for the hospital of $72,961 €$ during the study period. Considering the 15 two-stage revision cases, they also contributed to a slight increase on the overall hospital CMI by 0.00014 in 2014 and 0.00013 in 2015, which lead to an increase in overall hospital reimbursement of $100,173 €$ during the study period $(173,134 €$ for infected cases together).

\section{Discussion}

It is no surprise that PJI management represents a substantial clinical and economic burden for hospitals, health-care systems and, most importantly, patients. Notwithstanding, despite its increasing relevance, there are but a few papers that debate this issue. As far as we are aware this is the first study that analyzes the economic impact of prosthetic joint infection within the Portuguese public health system.

The full economic impact of PJI is ultimately much greater than the sum of all available parcels that can be objectively ascertained. Together with the commonly reported direct in-hospital costs, there are also less commonly reported outpatient costs and even indirect costs that are virtually impossible to assess, such as productivity loss and absenteeism from work of the patient or even his caregivers. Comparison between different papers is also made extremely difficult because of dramatic differences from one setting to another depending on the type of healthcare system and corresponding nation-specific economic standards.

In a paper by Kurtz et al [2] including over 150,000 PJI cases, the authors found that the average total hospital costs for infected hip revision were $\$ 72,700$ United States dollars (USD) in 2001 and $\$ 93,600$ USD in 2009. The average charges for infected knee revision were $\$ 58,700$ USD in 2001 and $\$ 74,900$ USD in 2009. Treatment costs for PJI knee patients were generally lower than in the hip with an average difference of \$5,965 USD. More recent studies from the US include not only inpatient but also outpatient services costs. In 2014, Kapadia et al[6] identified 21 infected TKA and matched them to 21 non-infected patients who underwent uncomplicated primary surgery. Naturally, patients with PJI had significantly longer hospitalizations, more readmissions and more clinic visits. The mean total episode cost (fixed- and variable-direct costs) for patients with a surgical site infection was $\$ 116,383$ USD (range, $\$ 44,416$ to $\$ 269,914)$ which was significantly higher than a mean $\$ 28,249$ USD(range, $\$ 20,454$ to $\$ 47,957$ ) in the matched group. Just recently, the same authors studied 16 consecutive infected THA and matched them to 32 non-infected patients. Similarly, the mean episode cost, was significantly higher in the infected group, $\$ 88,623$ USD (range, \$44,043-\$158,202) when compared to the matched cohort, $\$ 25,659$ USD (range, $\$ 13,595-\$ 48,631)[7]$.

Fernandez-Fairen et al [8] performed a systematic review of the literature and naturally found huge discrepancies in absolute values between publications according to its country of origin. Nonetheless, the cost for septic revision was consistently around 2-4 times more expensive than 
primary surgery. It was also 1.5-3 times more expensive than aseptic revision surgery.

Still, some PJI cases can be treated effectively without revision surgery. An Australian study by Peel et al[9], aimed specifically to calculate the cost associated with debridement and implant retention. For that, they focused on 21 prosthetic joint infections (12 THA and 9 TKA) and matched them to 42 control patients with uneventful primary joint replacements. They included inpatient expenses (human resources, operating theater, prosthesis, medical imaging, pathology, pharmacy, etc.) but also outpatient expenses including readmissions but also follow-up medical and nursing visits, medical imaging, pathology and pharmacy (including dispensed antibiotics). For patients with infection the total cost was $\$ 69,414$ Australian dollars (AUD) compared with $\$ 22,085$ AUD in controls, with significant differences across almost all areas of patient care. The cost of infection including index operation and costs of PJI management was 3.1 times the cost of uneventful primary arthroplasty in this study.

Our study is unique since it examines the costs of different treatment options (DAIR or revision surgery), plus it compares them with a group of uncomplicated primary arthroplasties and aseptic revision surgery that serve as reference for costs comparison. Directly comparing absolute values between countries is unfair as economic environment are extremely different. Although Portugal spends $8.9 \%$ of its gross domestic product (GDP) on health systems, which is only $0.1 \%$ less than average across Organization for Economic Co-operation and Development (OECD) countries, it has one of the lowest GDP and mean salaries in Europe[10]. As such, the authors believe that a fairer comparison of the cost of PJI and its treatment alternatives is to use the cost of uneventful primary arthroplasty as a reference measure.

There are of course limitations to our study. Our analysis focuses exclusively on direct in-hospital costs and does not consider outpatient direct costs like the money spent on antibiotics, consultations and returns to the emergency room or indirect costs. Also, ours is a single center study. While this design is advantageous to ensure complete economic data for all patients included, it does not take into consideration heterogeneity in costs or even treatment approaches between institutions.

Nevertheless, results of the current study highlight the substantial direct costs of prosthetic joint infection. It is clear that, even when infection is timely diagnosed and treated with debridement, appropriate antibiotics and implant retention, it is an expensive complication, more than doubling the costs of uncomplicated arthroplasty. In chronic infections requiring a two-stage exchange revision, costs rise to about 3.5 times that of a primary arthroplasty and 1.5 times that of an aseptic revision.

Knowledge of the costs associated with treatment of PJI is a critical part of optimizing existing resources in this era of worldwide financial concerns. The cost effectiveness of newer prophylactic measures can only be appreciated by knowing the real cost of infection. A major future challenge will be to define the role of different treatment strategies (DAIR, one-stage or two-stage revision surgery) taking in consideration the cost-effective perspective.

Another major economic implication of PJI associated costs is how to organize infection care. It is widely recognized that dedicated specialized centers are able to obtain better results, probably in a more cost-effective way. In many health systems such as the Portuguese public health system, a major obstacle to the creation of such reference centers is the belief that there is a lack of adequate compensation for the treatment of infected cases thereby limiting management willingness to treat external cases. Findings of other European healthcare systems with a reimbursement methodology based on the DRG system such as Germany suggests this belief to be true as costs exceed financial receipts in infected cases [11].

Our specific reimbursement methodology, as described previously in the methods section, adds another major variable. Although direct compensation for treating infected cases is much lower than calculated costs, infected cases do push the overall Hospital CMI slightly upwards thus increasing financial compensation for the entire cohort of treated patients.

This small but tangible effect, seems to mitigate the apparent negative financial impact on the Orthopedic Department by increasing compensation for the entire cohort of patients treated within the entire hospital.

In brief, the findings of this study may serve as a useful tool to more accurate resource allocation in PJI prophylaxis and management. Also, while they lack confirmation in larger cohorts as well as different treatment modalities such as one-stage revision surgery, our results seem to suggest the creation of a specialized center willing to receive external referred patients may not be as economically detrimental to the institution as previously thought.

\section{Acknowledgments}

The authors would like to thank Professor António Oliveira, Head of Orthopedic Department for allowing us access to all the data presented and help 
in better understanding national health system and institutional reimbursement policies.

\section{Competing Interests}

The authors have declared that no competing interest exists.

\section{References}

1. Dale H, Fenstad A, Hallan G, Havelin LI, Furnes O, Overgaard S, et al. Increasing risk of prosthetic joint infection after total hip arthroplasty. Acta Orthop. 2012;83(5): 449-58

2. Kurtz SM, Lau E, Watson H, Schmier JK, Parvizi J. Economic burden of periprosthetic joint infection in the United States. J Arthroplasty. 2012;27(8 Suppl): 61-5.

3. Huotari K, Peltola M, Jamsen E. The incidence of late prosthetic joint infections: a registry-based study of 112,708 primary hip and knee replacements. Acta Orthopaedica. 2015; 86 (3): 321-5.

4. Kurtz SM, Ong KL, Lau E, Bozic KJ. Impact of the economic downturn on total joint replacement demand in the United States: updated projections to 2021. J Bone Joint Surg Am. 2014;96(8): 624-30.

5. Patel A, Pavlou G, Mujica-Mota RE, Toms AD. The epidemiology of revision total knee and hip arthroplasty in England and Wales: a comparative analysis with projections for the United States. A study using the National Joint Registry dataset. Bone Joint J 2015;97-B(8): 1076-81.

6. Kapadia BH, McElroy MJ, Issa K, Johnson AJ, Bozic KJ, Mont MA. The economic impact of periprosthetic infections following total knee arthroplasty at a specialized tertiary-care center. J Arthroplasty. 2014;29(5): 929-32.

7. Kapadia BH, Banerjee S, Cherian JJ, Bozic KJ, Mont MA. The Economic Impact of Periprosthetic Infections After Total Hip Arthroplasty at a Specialized Tertiary-Care Center. J Arthroplasty. 2016;31(7): 1422-6.

8. Fernandez-Fairen $\mathrm{M}$, Torres A Menzie A Hernandez-Vaquero D, Fernandez-Carreira JM, Murcia-Mazon A, et al. Economical analysis on prophylaxis, diagnosis, and treatment of periprosthetic infections. Open Orthop J. 2013;7: 227-42.

9. Peel TN, Dowsey M, Buising KL, Liew D, Choong PFM. Cost analysis of debridement and retention for management of prosthetic joint infection. Clin Microbiol Infect. 2013;19(2): 181-6.

10. OECD. Health at a Glance 2017: OECD Indicators. OECD Publishing, Paris. 2017: http://dx.doi.org/10.1787/health_glance-2017-en.

11. Haenle M, Skripitz C, Mittelmeier W, Skripitz R. Economic impact of infected total knee arthroplasty. ScientificWorldJournal. 2012; 2012: 196515. 